\title{
Teaching Video NeuroImages: Cerebellar esotropia
}

\section{A pitfall in ophthalmology and neurology}

Anne-Catherine Chapelle, MD, Gordon T. Plant, MD, PhD, and Diego Kaski, MD, PhD

Neurology ${ }^{\circledR}$ 2019;93:e114-e115. doi:10.1212/WNL.0000000000007729

Figure T1-weighted axial MRI shows a type 1 Chiari malformation

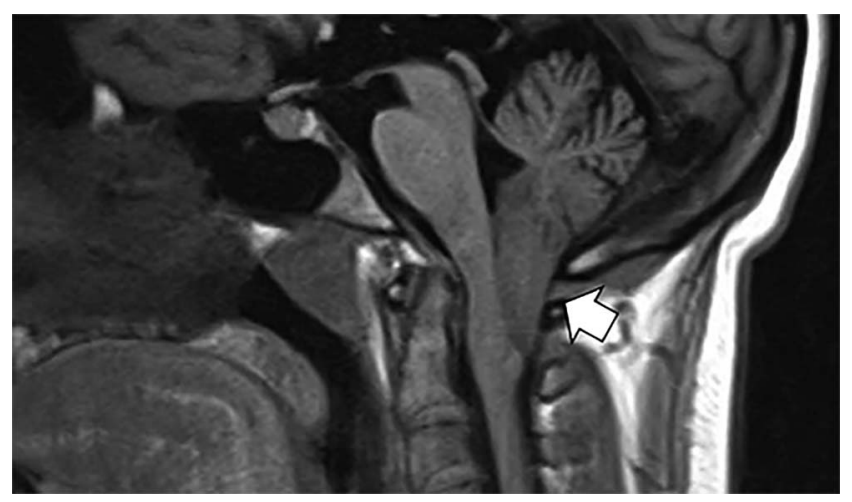

A 17-year-old girl presented with slowly progressive binocular horizontal diplopia, worse for distance viewing. She had distance esotropia (video), gaze-evoked nystagmus, broken smooth pursuit, reduced horizontal optokinetic nystagmus, and impaired vestibulo-ocular reflex suppression (VORS; video), in keeping with cerebellar dysfunction. The patient had full abduction during monocular viewing and head rotations, and normal saccadic velocities, excluding a bilateral sixth nerve palsy. An MRI scan confirmed a type 1 Chiari malformation (figure). A careful oculomotor assessment in patients with esotropia, including bedside VORS, ${ }^{1}$ may identify cerebellar signs that would suggest the tropia is cerebellar in origin. ${ }^{2}$ Cerebellar esotropia may arise from dorsal vermis impairment, ${ }^{2}$ or possibly from floccular and parafloccular dysfunction, explaining the associated abnormalities in smooth pursuit, optokinetic nystagmus, and VORS that share common neural pathways.

\section{Study funding}

Not targeted funding reported.

\section{Disclosure}

The authors report no disclosures relevant to the manuscript. Go to Neurology.org/N for full disclosures.
Correspondence

Dr. Kaski

d.kaski@ucl.ac.uk

MORE ONLINE

- Video

$\rightarrow$ Teaching slides

links.lww.com/WNL/

A914 


\section{Appendix Authors}

\begin{tabular}{|c|c|c|c|}
\hline Name & Location & Role & Contribution \\
\hline $\begin{array}{l}\text { Diego } \\
\text { Kaski, MD }\end{array}$ & $\begin{array}{l}\text { University College } \\
\text { London, UK }\end{array}$ & Author & $\begin{array}{l}\text { Designed and } \\
\text { conceptualized study, } \\
\text { drafted the manuscript for } \\
\text { intellectual content, video } \\
\text { editing, approved final } \\
\text { version }\end{array}$ \\
\hline $\begin{array}{l}\text { Anne- } \\
\text { Catherine } \\
\text { Chapelle, } \\
\text { MD }\end{array}$ & $\begin{array}{l}\text { Centre Hospitalier, } \\
\text { University of Liege, } \\
\text { Belgium }\end{array}$ & Author & $\begin{array}{l}\text { Drafted the manuscript } \\
\text { for intellectual content, } \\
\text { video acquisition, } \\
\text { approved final version }\end{array}$ \\
\hline $\begin{array}{l}\text { Gordon } \\
\text { Plant, MD }\end{array}$ & $\begin{array}{l}\text { National Hospital } \\
\text { for Neurology and } \\
\text { Neurosurgery, UK }\end{array}$ & Author & Approved final version \\
\hline
\end{tabular}

\section{References}

1. Zee DS. Suppression of vestibular nystagmus. Ann Neurol 1977;1:207.

2. Wong SH, Patel L, Plant GT. Acquired esotropia in cerebellar disease: a case series illustrating misdiagnosis as isolated lateral rectus paresis and progression over time. Neuro-ophthalmology 2015;39:59-63. 


\section{Neurology}

\section{Teaching Video NeuroImages: Cerebellar esotropia: A pitfall in ophthalmology and neurology}

Anne-Catherine Chapelle, Gordon T. Plant and Diego Kaski

Neurology 2019;93; e114-e115

DOI 10.1212/WNL.0000000000007729

\section{This information is current as of July 1, 2019}

\section{Updated Information \& Services}

References

Subspecialty Collections

Permissions \& Licensing

Reprints including high resolution figures, can be found at: http://n.neurology.org/content/93/1/e114.full

This article cites 2 articles, 0 of which you can access for free at: http://n.neurology.org/content/93/1/e114.full\#ref-list-1

This article, along with others on similar topics, appears in the following collection(s):

All Clinical Neurology

http://n.neurology.org/cgi/collection/all_clinical_neurology All Neuro-ophthalmology

http://n.neurology.org/cgi/collection/all_neuroophthalmology

All Neurotology

http://n.neurology.org/cgi/collection/all_neurotology

Diplopia (double vision)

http://n.neurology.org/cgi/collection/diplopia_double_vision MRI

http://n.neurology.org/cgi/collection/mri

Information about reproducing this article in parts (figures,tables) or in its entirety can be found online at:

http://www.neurology.org/about/about_the_journal\#permissions

Information about ordering reprints can be found online: http://n.neurology.org/subscribers/advertise

Neurology ${ }^{\circledR}$ is the official journal of the American Academy of Neurology. Published continuously since 1951, it is now a weekly with 48 issues per year. Copyright (O 2019 American Academy of Neurology. All rights reserved. Print ISSN: 0028-3878. Online ISSN: 1526-632X.

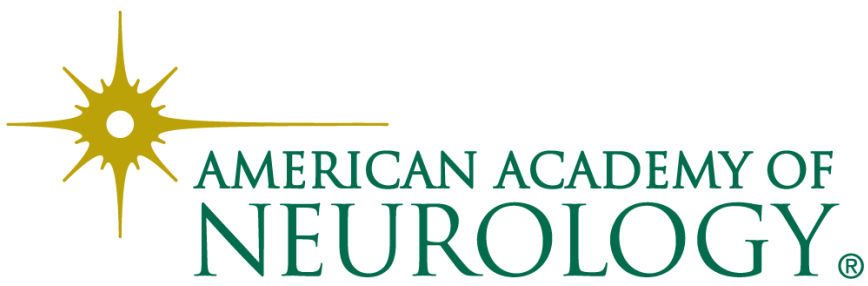

